\title{
MNU Induction of Neoplasia in a Platyfish Model
}

\author{
Steven Kazianis, Irma Gimenez-Conti, Richard B. Setlow, Avril D. Woodhead, \\ John C. Harshbarger, David Trono, Mark Ledesma, Rodney S. Nairn, and \\ Ronald B. Walter
}

\begin{abstract}
Department of Chemistry and Biochemistry (SK, ML, RBW), Southwest Texas State University, San Marcos, Texas; Department of Carcinogenesis, Science Park-Research Division, M. D. Anderson Cancer Center, University of Texas, Smithville, Texas (IG-C, DT, RSN); Department of Biology, Brookhaven National Laboratory, Upton, New York (RBS, ADW); and Department of Pathology (JCH), George Washington University Medical Center, Washington, D.C.
\end{abstract}

\begin{abstract}
SUMMARY: Interspecific hybrid crosses between members of the fish genus Xiphophorus have been used for over 70 years to study the genetic aspects of melanoma formation. In the well-established "Gordon-Kosswig" cross, the platyfish X. maculatus is outcrossed to the swordtail $X$. helleri, and the resulting backcross segregants spontaneously develop melanoma. We recently produced a distinct cross between $X$. maculatus and another platyfish species, $X$. couchianus. $X$. maculatus strain Jp $163 \mathrm{~A}$ is homozygous for several X-linked pigment pattern genes, including the Spotted dorsal (Sd), Dorsal red (Dr), and Anal fin spot (Af). Af is a sex-limited trait, coding exclusively for melanophores distributed on the modified anal fin or "gonopodium" in the adult male fish. Within $\mathrm{F}_{1}$ and $\mathrm{BC}_{1}$ hybrids (to $X$. couchianus), the $\mathrm{Sd}$ pigment pattern is phenotypically suppressed, whereas Dr and Af are enhanced. We exposed $\mathrm{BC}_{1}$ hybrids to the direct-acting carcinogen $N$-methyl- $N$-nitrosourea (MNU). Treatment led to the development of schwannomas, fibrosarcomas, and retinoblastomas. In addition, numerous MNU-treated males that inherited $A f$ developed a pronounced melanotic phenotype, with melanin-containing cells oftentimes totally covering the gonopodium and extending further to grow within the ventral regions of the fish. Genetic linkage analysis of the $\mathrm{BC}_{1}$ hybrids revealed a significant $(p<0.01)$ association between CDKN2X genotype and the phenotypic degree of melanization. Such an association is consistent with a locus within linkage group $V$ playing a role in the development of melanosis and delineates three genetic preconditions and a carcinogenic scheme resulting in melanosis of the ventral regions of hybrid fish. The overall study further alludes to the potential of using Xiphophorus fish to study carcinogenic mechanisms for tumors other than melanoma (schwannoma, fibrosarcoma, and retinoblastoma) and should enable extensive pathologic and molecular genetic studies of derived neoplastic abnormalities. (Lab Invest 2001, 81:1191-1198).
\end{abstract}

$A$ nimal models are an invaluable tool in understanding the complexities of neoplastic disease. Interspecific hybrid crosses between members of the fish genus Xiphophorus (currently comprising 22 species) have been used for over 70 years to study the genetic aspects of melanoma formation (Gordon, 1931a; Kosswig, 1927; Nairn et al, 1996b; Schartl, 1995). Typically, genetic traits, such as melanistic pigment patterns, are well regulated in the parental strains, but are inadequately regulated in hybrid fish, resulting in uncontrolled proliferation, and ultimately, in the development of neoplastic exophytic growths such as nodular melanomas. Numerous crosses between parental strains have generated hybrid fish that

Received March 8, 2001

This study was supported by Grant RR12253 from the National Center for Research Resources, Grant ATP-030 from the Texas Higher Education Coordinating Board, United States Public Health Service Grants CA09480, CA55245, CA75137, CA76693, and ES07784. The research at Brookhaven National Laboratory was performed under the auspices of the U.S. Department of Energy under Contract No. DE-AC02-98CH10886.

Address reprint requests to: Dr. Ronald B. Walter, Department of Chemistry and Biochemistry, Southwest Texas State University, 601 University Drive, San Marcos, TX, 78666. E-mail: RW12@swt.edu develop melanomas with great predictability, both for anatomical position and age of onset. Melanomas derived within such hybrid crosses are clearly genetically predetermined, as was demonstrated for several Xiphophorus tumor models (Nairn et al, 1996b; Schartl, 1995; Vielkind et al, 1989). A candidate tumor suppressor gene, CDKN2X, which maps to Xiphophorus linkage group V (LG V) within a genomic region implicated in melanoma tumor suppression and pigment pattern regulation, was cloned (Kazianis et al, 1998, 1999; Siciliano et al, 1976; Vielkind, 1976). This gene shows structural similarity to the human CDKN2A (P16) locus, which is presently thought to be involved in suppressing human melanoma and other tumors as well (Chin et al, 1998; Ruas and Peters, 1998).

$N$-methyl- $N$-nitrosourea (MNU) is an alkylating agent that methylates DNA bases at nucleophilic sites (generating $\mathrm{N}^{7}$ and $\mathrm{N}^{3}$ alkylpurines). The primary mutagenic lesion is believed to be $\mathrm{O}^{6}$ methylguanine (Friedberg et al, 1995). MNU induces numerous cancers in rodents, including mammary carcinomas and thyroid tumors in rats (Ohshima and Ward, 1984; Zarbl et al, 1985), as well as thymic lymphomas in mice (Frei and Lawley, 1980; Joshi and Frei, 1970; Richie et al, 
1996). This complete carcinogen also effectively induced a wide array of tumors in Xiphophorus hybrids, including neuroblastomas, melanomas, and fibrosarcomas, in addition to rhabdomyosarcomas and various carcinomas, which occurred at a much reduced incidence (Schwab et al, 1978a, 1978b, 1979). The development of neuroblastomas was strongly associated with one particular hybrid cross involving $X$. variatus and $X$. helleri, and not in 64 other nonhybrid species/strains and derived hybrids (Schwab et al, 1978a, 1978b, 1979).

We recently generated a cross between $X$. maculatus and another platyfish species, $X$. couchianus (Fig. 1). X. maculatus strain Jp $163 \mathrm{~A}$ is homozygous for a macromelanophore pigment pattern gene called Spotted dorsal $(S d)$ and a linked pterinophore locus called Dorsal red (Dr; Fig. 2A). Both loci are linked on the $\mathrm{X}$ chromosome and recombination between them has been rarely reported (Kallman, 1975; Kallman and Schreibman, 1971). The homologous $X$. couchianus sex chromosomes lack both $S d$ and $\mathrm{Dr}$ loci. $\mathrm{F}_{1}$ hybrids and subsequently produced $\mathrm{BC}_{1}$ hybrids show a pronounced phenotypic suppression of Sd, but an en-

\section{X. maculatus Jp 163 A}
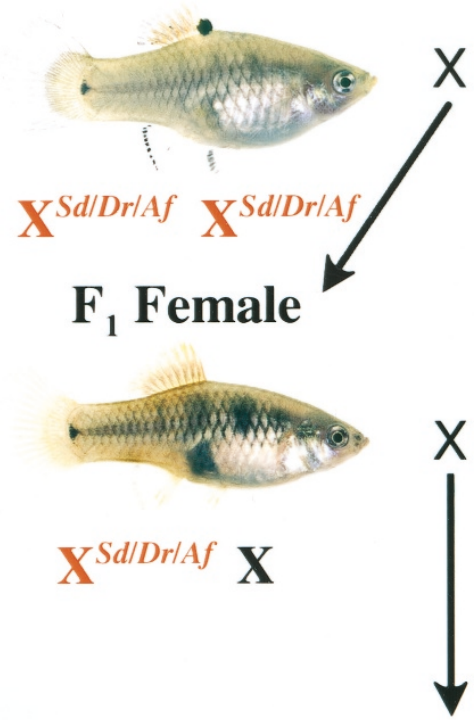

hancement of Dr. The suppression of the Sd pigment pattern is a striking contrast to the severe enhancement of the Spot-sided (Sp) pigment pattern in a parallel genetic cross with the same parental species, but using $X$. maculatus strain Jp 163 B (Nairn et al, 1996b; Setlow et al, 1993, 1989). In addition, the Sd pigment pattern itself, derived from $X$. maculatus strain Jp $163 \mathrm{~A}$, is typically enhanced in numerous genetic crosses, including hybrids between $X$. maculatus and $X$. helleri. Within this well-studied model, referred to as the "Gordon-Kosswig" cross, the Sd pigment pattern is enhanced to the point of extreme melanosis and development of malignancy (Morizot and Siciliano, 1983; Schartl, 1995; Vielkind, 1976).

We exposed numerous $\mathrm{BC}_{1}$ individuals derived from the hybrid cross between the two platyfish species, $X$. maculatus and $X$. couchianus, to aqueous solutions containing the direct-acting carcinogen MNU. Melanotic hyperplasia (melanosis), retinoblastomas, fibrosarcomas, and schwannomas were produced; their incidence and histological properties are described.

\section{X. couchianus}

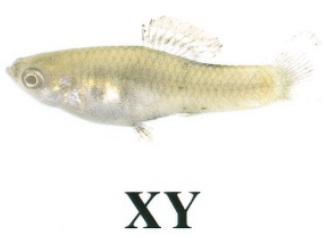

\section{X. couchianus}

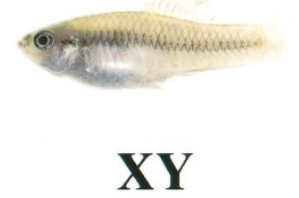

\section{Backcross hybrids}
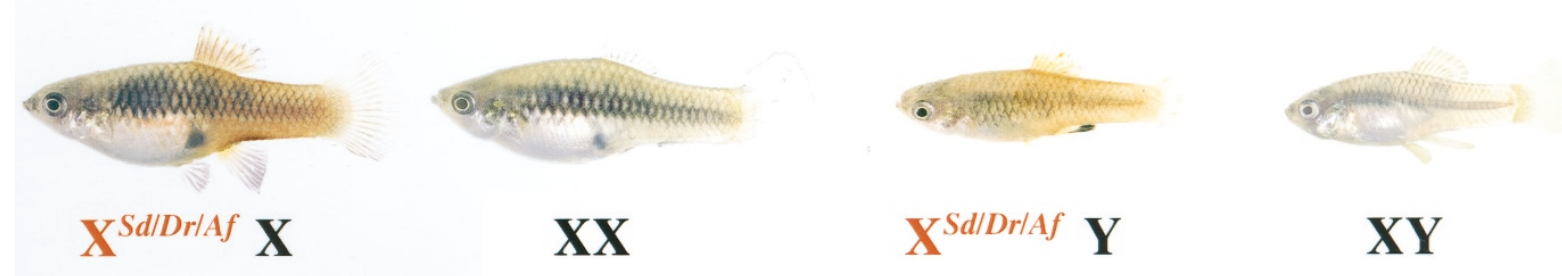

Figure 1.

Generalized depiction of the (Xiphophorus maculatus Jp $163 \mathrm{~A} \times X$. couchianus) $\times X$. couchianus cross. A $X$. maculatus individual, homozygous for the $S d$ macromelanophore pattern locus and the pterinophore locus $D r$, is mated to a $X$. couchianus lacking both loci. The resulting $F_{1}$ hybrids show a phenotypic suppression of $\mathrm{Sd}$ but an enhancement of $\mathrm{Dr}$. This pattern of respective suppression and enhancement is preserved in the $\mathrm{BC}_{1}$ hybrid progeny. Within male offspring, the sex-limited pigment pattern locus $A f$ is inherited and expressed by half of the male progeny. This pigment pattern is carried by the $\mathrm{X}$ chromosome derived from $X$. maculatus and is also not present in $X$. couchianus. For simplicity, only the genotypes for $X$. maculatus are depicted. 
A
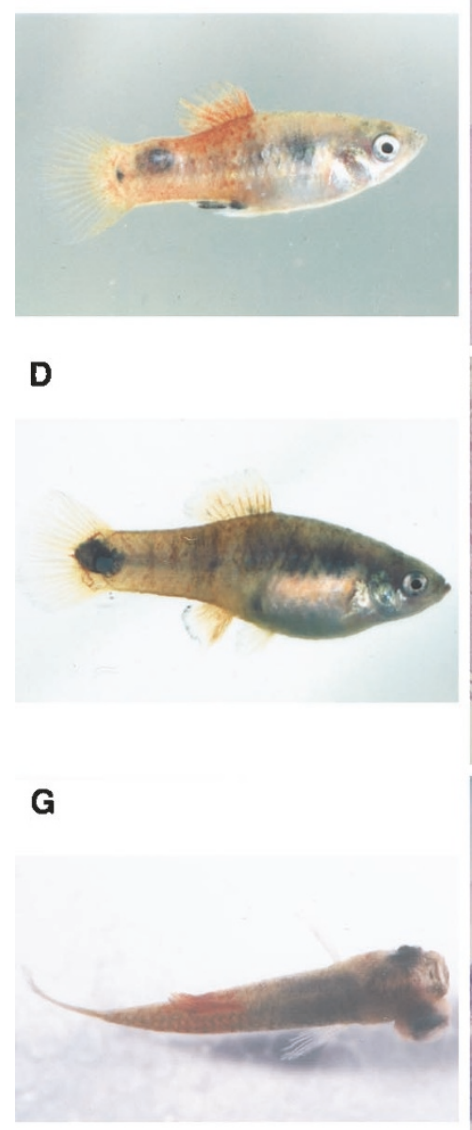

Figure 2.

Development of schwannomas, fibrosarcomas, and retinoblastomas in $\mathrm{N}$-methyl- $\mathrm{N}$-nitrosourea (MNU)-treated fish. $\mathrm{A}, \mathrm{BC}_{1}$ hybrid fish showing development of a schwannoma on the caudal peduncle. B, A well-circumscribed schwannoma derived from the fish in A. C, Area of typical palisades. D, Example of a fish developing fibrosarcoma on the base of the caudal fin. E, Low-magnification image of a fibrosarcoma infiltrating muscular structure. F, Muscle bundles compressed by whorled masses of densely packed spindle cells. G, Photograph of a fish taken from above showing protrusion of the right eye. $\mathrm{H}$, The retinoblastoma in the fish depicted in $\mathrm{G}$, with tumor cells invading the inner and outer surface of the retina, the sclera, and the optic nerve. I, Higher magnification photomicrograph showing tumor cell histological characteristics. The arrows delineate the area that shows a rosette-like arrangement. Scale bars are $200 \mu \mathrm{m}$.

\section{Results and Discussion}

\section{General Description of the Animal Model}

$\mathrm{BC}_{1}$ hybrid fish were produced between $X$. maculatus and $X$. couchianus, using either $F_{1}$ hybrid females or males. Figure 1 depicts one crossing scheme used to generate such hybrid fish, while following the inheritance of the sex chromosomes which harbor the Sd macromelanophore locus, the Dr pterinophore locus (Kallman, 1975), and a pigment pattern locus referred to as Anal fin spot (Af, also referred to as Anal fin black [Ab]) (Gordon, 1931b; Schartl et al, 1995). The Sd and $\mathrm{Dr}$ loci are tightly linked on the $\mathrm{X}$ chromosome of $X$. maculatus, and recombination is rare (Kallman, 1975). Within the $\mathrm{F}_{1}$ and $\mathrm{BC}_{1}$ hybrids to $X$. couchianus, the $\mathrm{Dr}$ pigment pattern is sharply overexpressed, so that the fish are distinctly orange in color. Pterinophore cells that are usually restricted to the dorsal fin in the parental $X$. maculatus strain Jp 163 A are widespread over the body, including the trunk. In contrast, the Sd pigment pattern is phenotypically suppressed, be- cause only $13.6 \%$ (21/154) Dr-bearing backcross hybrids also phenotypically express $\mathrm{Sd}$.

A total of $276 \mathrm{BC}_{1}$ fish were raised with $171 \mathrm{BC}_{1}$ individuals used as untreated controls. Treatment of $105 \mathrm{BC}_{1}$ fish with the direct-acting carcinogen MNU yielded several types of neoplasms, including schwannomas, fibrosarcomas, and retinoblastomas (Table 1). None of the control fish developed any discernible neoplasms. In addition, MNU treatment resulted in numerous individuals with melanosis. The histopathological features of these abnormalities are described below.

\section{Development of Schwannomas, Fibrosarcomas, and Retinoblastomas in MNU-Treated Fish}

Schwannomas are benign tumors composed entirely of Schwann cells, which are derived from the neurilemma of a nerve fiber. Schwannomas developed at an incidence of $2.8 \%(3 / 105)$ and were typically found on the caudal peduncle region of the fish (between the 
Table 1. Incidence of Lesions in $\mathbf{B C}_{1}$ Fish Treated with $\mathrm{MNU}^{\mathrm{a}}$

\begin{tabular}{lcr}
\hline \multicolumn{1}{c}{ Lesion } & $\begin{array}{c}\text { MNU } \\
(n=105)\end{array}$ & $\begin{array}{r}\text { MNU } \\
(\%)\end{array}$ \\
\hline Schwannoma & 3 & 2.8 \\
Fibrosarcoma & 7 & 6.6 \\
Retinoblastoma & 4 & 3.8 \\
Unrestricted melanosis $^{b}$ & 11 & 10.4 \\
\hline
\end{tabular}

MNU, $N$-methyl- $N$-nitrosourea.

a Five-week-old fish exposed to $1.0 \mathrm{mM}, 2$ hours, on Days 0,2 , 4, and 6. No neoplasms occurred in 171 untreated control fish.

${ }^{b}$ Unrestricted melanosis is defined as aggregations of melanin containing cells that are found outside the confines of the gonopodium. Typically, such cells grow within the integument of the ventral and mid-ventral flanks of the fish (see text and Fig. 3).

dorsal and caudal fins). All were unilateral, wellcircumscribed, whitish lesions with globular configurations (Fig. 2A). Microscopically, the tumors were encapsulated by dense bundles of muscle (Fig. 2B). Some areas exhibited compact fascicular tissue (Antony A pattern) and typical palisades resulting from stacked arrays of nuclei alternating with nuclear fibrillar zones composed of cell processes (Fig. 2, B and C). Mitotic figures were absent.

Fibrosarcomas are malignant neoplasms derived from mesenchymal cells. Fibrosarcomas were typically found on the caudal peduncle area (Fig. 2D), although several growths also occurred elsewhere, such as on the operculum and the dorsal trunk above the midlateral line. They developed at an incidence of $6.6 \%(7 / 105)$, and several individuals displayed multiple exophytic tumors dispersed throughout the body surface. Microscopic examination showed packed spindle-shaped fibroblasts infiltrating surrounding structures (Fig. 2E). Muscle bundles were compressed by whorled masses of densely packed spindle cells (Fig. 2F).

Retinoblastomas are an intraocular malignant neoplasm derived from embryonic neuronal cells. Retinoblastomas were harvested when protrusion of the eye was evident (Fig. 2G) and observed in 3.8\% $(4 / 105)$ of the MNU-treated $\mathrm{BC}_{1}$ hybrids. The retinoblastomas were all unilateral. Microscopic examination revealed tumor cells resembling neuroblasts that were invading the inner and outer surface of the retina, the sclera, and the optic nerve. In one case, the tumorous cells had spread along the optic nerve to the brain (Fig. 2H). In another individual, tumor cells were found in the middle part of the head invading the muscle associated with the opposite eye. The tumors were intensely cellular and densely packed, and, in some instances, round neoplastic cells with hyperchromatic nuclei, scant cytoplasm, and abundant mitoses were randomly distributed (Fig. 2I). Moderately differentiated rosette-like structures were distinguishable in two of the neoplasms (Fig. 2l, arrows). Generally, all the retinoblastomas were well vascularized.

\section{Development of Melanosis after MNU Treatment}

Analysis of phenotypic segregation data within $\mathrm{BC}_{1}$ hybrids (generated using both $\mathrm{F}_{1}$ males and females) revealed that the Af pigment pattern locus resides on the $X$ chromosome derived from $X$. maculatus. This gene is linked to $D r$ with an estimated recombination rate of approximately $3.4 \%$ (2 recombinants within 58 informative fish). Within the $X$. maculatus strain Jp 163 A, Af codes for melanophores distributed within the modified anal fin or "gonopodium" exclusively within adult male fish; thus, it is a sex-limited trait. The melanic cells are usually restricted toward the tip of the gonopodium, and most are concentrated on the most ventral fin rays (Fig. $3 A$ ). Unique to the $F_{1}$ and $\mathrm{BC}_{1}$ hybrids described herein, Af is phenotypically enhanced, and in many cases melanic cells are distributed along the entire length of the gonopodium. $X$. couchianus, the other species used, does not harbor the Af pigment pattern.

In MNU-treated fish, all pigmented lesions were derived from the Af pigment pattern, with melanincontaining cells oftentimes totally covering the gonopodium and proceeding to penetrate into the ventral regions of the fish. Figure 3 depicts two exemplary $\mathrm{BC}_{1}$ fish that had been treated with MNU. In the first (Fig. 3B), the enhancement of Af was not pronounced, because melanization is restricted to the gonopodium. Histology showed pigmented melanocytes and melanophores located near and around the bony rays of the gonopodium (Fig. 3D). However, the second fish shown (Fig. 3C) had developed radially spreading melanosis derived from Af, and the cells had proliferated to encompass the neighboring pelvic fins and extended into the body. The pronounced melanosis was no longer restricted to the gonopodial region. However, the melanocytes and melanophores were restricted to the integument, exhibiting a distinct lack of invasiveness (Fig. 3E). Uniform, dense spindlepattern melanin pigment was distributed along and restricted to the dermis and basal layer of the gonopodial skin. No invasion of body tissues was detected in any of the restricted Af individuals. Ten additional such $\mathrm{BC}_{1}$ fish revealed similar pathological characteristics. The overall lack of invasiveness derived from Af is in stark contrast to the vast majority of melanotic neoplasms derived from Xiphophorus hybrid models, where invasion of underlying tissues and exophytic growths are commonly observed (Reed and Gordon, 1931; Schartl et al, 1995; Sobel et al, 1975).

\section{Genotypic Association of LG V and Melanosis}

Eleven of $28(39 \%)$ MNU-treated males that inherited Af developed a pronounced (ie, unrestricted) melanotic phenotypic enhancement similar to that in the individual shown in Figure 3C. All of them were genotyped as homozygotes for CDKN2X using the PCR (Mullis et al, 1986). In addition, we genotyped 9 of the individuals that had developed restricted pigmentation within the gonopodium, and 7 of them were heterozygous. Together, these results are sig- 

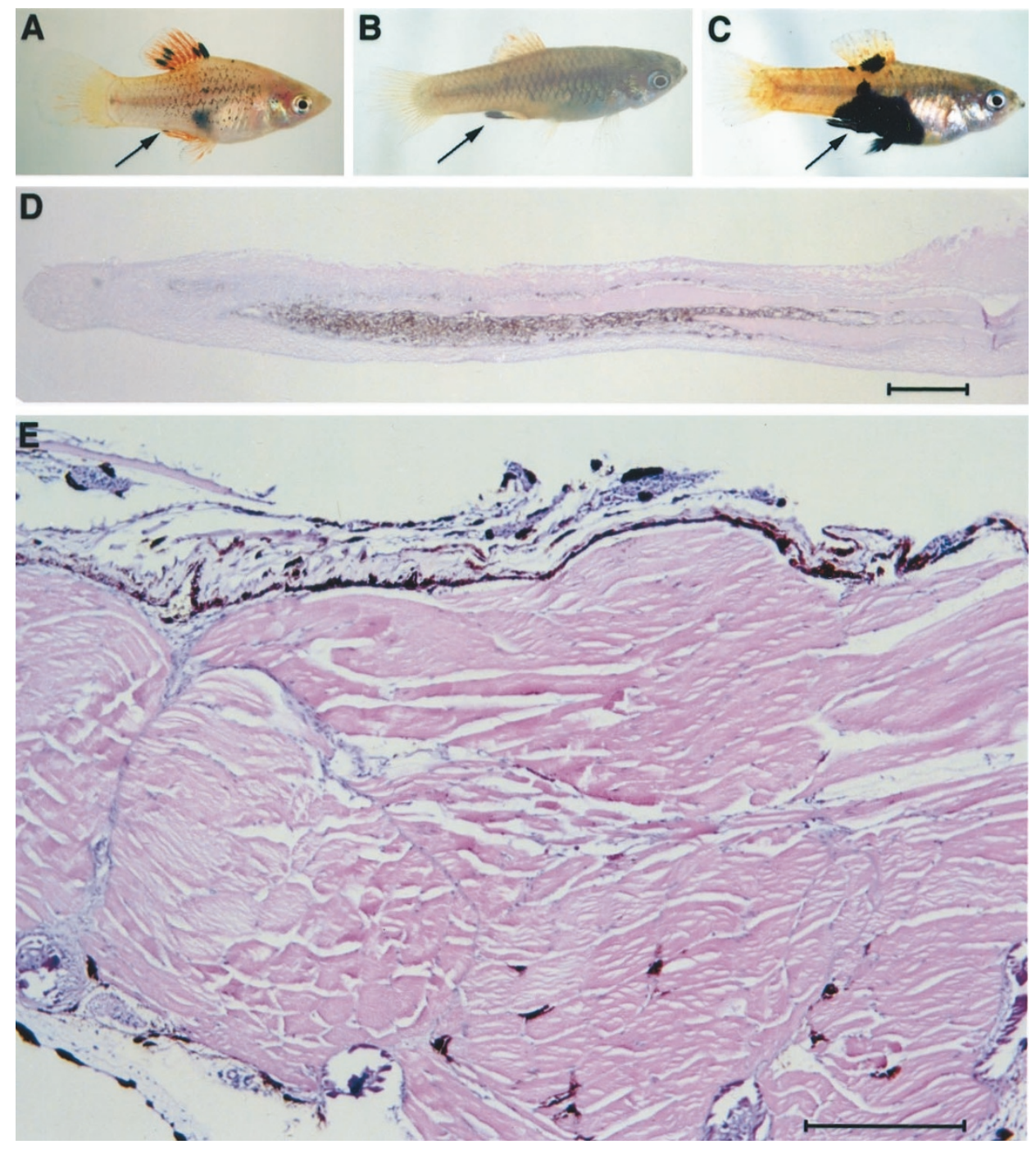

Figure 3.

Development of the Af pigment pattern and melanosis induced by MNU. A, A X. maculatus strain Jp $163 \mathrm{~A}$ male fish, showing the Af pigment pattern at the distal gonopodial region. The $\mathrm{Sd}$ pigment pattern is manifested by melanic spotting on the dorsal fin. $\mathrm{B}, \mathrm{BC}_{1}$ hybrid fish with Af melanization restricted to the gonopodium. $\mathrm{C}$, An MNU-treated fish with a radially spreading melanosis that is not restricted to the gonopodial region. D, Longitudinal section through the gonopodium showing melanization around the bony rays of the gonopodium. E, Cross section of the anterior caudal peduncle showing pronounced hyperpigmentation within the integument with a lack of invasiveness. Scale bars are $200 \mu \mathrm{m}$.

nificant $\left(\chi^{2}=12.8, p<0.01\right)$ because $C D K N 2 X$ genotypes correlate with the phenotypic development of restricted or unrestricted melanization derived from the Af pigment pattern (18 parentals, 2 recombinants, logarithm of odds [LOD] $=3.19$ ). Such a result is striking and consistent with a locus within LG V playing a role in the development of melanosis and being associated with the Af pigment pattern. There have been several previous reports (involving different genetic crosses and pigment patterns) of an association of Xiphophorus LG V genotypic data and melanoma formation and for spontaneous and UV-induced melanotic hyperplasia, although involvement with the Af pigment pattern has not been previously noted (Ahuja et al, 1980; Fornzler et al, 1991; Kazianis et al, 1998; Morizot and Siciliano, 1983; Siciliano and Wright, 1976).

\section{Summary}

MNU treatment of 105 hybrid fish derived from crossing two platyfish species resulted in the induction of several neoplasms at incidences between $2.8 \%$ and $6.6 \%$ (Table 1). These neoplasms did not develop in 171 control fish. In addition, $10.4 \%$ of treated backcross hybrids exhibited melanosis. We can clearly identify three genetic preconditions that must be met before melanosis develops in $\mathrm{BC}_{1}$ hybrids. Firstly, the individuals must inherit an $\mathrm{X}$ chromosome derived from the southern platyfish, $X$. maculatus, carrying a pigment pattern locus referred to as Af. Secondly, afflicted fish are always males, as the Af pigment pattern is sex-limited to them, and only such fish develop the restricted (to the gonopodium) or unrestricted melanosis. Thirdly, melanosis is significantly associated with the lack of inheritance of an autoso- 
mal locus derived from $X$. maculatus. Xiphophorus LG $V$ harbors a locus referred to as Diff which has been implicated in determining the state of differentiation of melanistic pigment cells (Ahuja et al, 1980; Siciliano et al, 1976; Vielkind, 1976). In addition, strong associations have been shown between LG V genotypes and spontaneous or UV-induced melanoma formation in several distinct Xiphophorus genetic crosses, all involving the platyfish $X$. maculatus (Kazianis et al, 1998). The tumor suppressor gene candidate CDKN2X maps to this autosomal region (Nairn et al, 1996a) and has shown strong genetic associations with melanotic phenotype and even stronger associations with melanoma formation (Kazianis et al, 1998; Nairn et al, 1996a). Thus, CDKN2X is considered a candidate for the classically defined Diff tumor suppressor gene (Kazianis et al, 1999). We examined BC hybrid male fish that had been exposed to MNU and had developed restricted or unrestricted melanistic pigmentation for CDKN2X alleles. The results significantly implicate this genomic region, because all $\mathrm{BC}_{1}$ fish developing unrestricted melanosis were homozygotes for $X$. couchianus CDKN2X alleles and lack $X$. maculatus genomic copies. Conversely, individuals not developing the unrestricted hyperpigmentation (even after MNU treatment) were heterozygous for CDKN2X. We are currently continuing studies of $C D K N 2 X$ and examining the genomic region encompassing this locus to address potential mechanisms leading to melanoma formation. Recent studies have indicated that CDKN2X allelic copies derived from differing species may show variable expression in cells of the melanocytic pathway, with the $X$. maculatus allele showing the highest expression in such cells (Kazianis et al, 1999, 2000). Such a simple mechanism may account for the development of melanoma in select crosses and is possibly responsible for the melanosis observed in the cross between $X$. maculatus and $X$. couchianus. We are actively investigating this mechanism and others to fully assess the role of CDKN2X in the cross between $X$. maculatus and $X$. couchianus, described herein.

In addition to the above-mentioned genetic preconditions, unrestricted melanosis is strongly associated with MNU treatment, because untreated individuals $(n=171)$ did not develop this phenotype. Hypothetically, MNU is acting on at least one other genetic locus that is expected to have antiproliferative properties. Such a gene would be a mutational target in MNU-treated animals in order for melanosis to become established, but the consequences would only be manifested in fish of a specific genetic heritage.

The discovery of schwannomas, fibrosarcomas, and retinoblastomas in the $\mathrm{BC}_{1}$ hybrids, albeit at a lower incidence, opens the additional possibility of studying the underlying genetic background necessary for induced development of these neoplasms as well. In addition, we may be able to identify the genomic targets after MNU treatment. We are currently exploring this, using hybrids between $X$. maculatus and $X$. couchianus, and in several other crosses. In general, the use of small aquarium teleosts, such as the genus Xiphophorus, zebrafish, and Japanese medaka, should prove extremely fruitful in the study of inducible neoplasia.

\section{Materials and Methods}

\section{Experimental Animals and MNU Treatment}

Animal care was performed in accordance with institutional guidelines. Parental stocks and hybrids used in this project were derived from the Xiphophorus Genetic Stock Center (Southwest Texas State University, San Marcos, Texas). Female X. maculatus (strain Jp 163 A, Rio Jamapa, Mexico) individuals, homozygous for the X-chromosomal Sd and $\mathrm{Dr}$ pigment patterns, were mated to $X$. couchianus males (Huasteca Canyon, Mexico). Both male and female $F_{1}$ hybrids were subsequently crossed to $X$. couchianus to produce $\mathrm{BC}_{1}$ hybrids. Artificial insemination protocols were not necessary to produce the $F_{1}$ and $B_{1}$ fish.

MNU was stored in ISO-PAC vials (Sigma, St. Louis, Missouri) and frozen until use. All treatments were carried out in glass beakers under fume hoods to minimize contamination. Five-week old fish were caught in disposable nets and transferred to $1.5 \mathrm{~L}$ clean (filtered) aquarium water within $2.0 \mathrm{~L}$ beakers. Freshly prepared $100 \mathrm{~mm}$ MNU solution $(\mathrm{pH} 4.5$, citrate buffer) was appropriately diluted to $1.0 \mathrm{~mm}$ (final concentration) and added to the beakers containing the fish. After a 2-hour exposure, the fish were rinsed in MNU-free aquarium water and returned to their home aquaria (5- to 30-gallon tanks). This exposure was repeated every other day, with up to four treatments. The survival rate at 2 months post-exposure was $87.7 \%$.

\section{Euthanasia of Fish and Processing for Histology}

$\mathrm{BC}_{1}$ hybrid fish usually were killed at 14 months of age unless accelerated development of a tumor required earlier euthanasia. They were scored for pigmentation phenotypes, sex, standard length, and the development of abnormal external growths. Euthanasia was performed with the commonly used anesthetic MS222 (tricane methane sulfate) diluted to an aqueous solution of $0.06 \%$. All lesions, along with surrounding normal tissues were excised and then fixed in $10 \%$ buffered formalin. After paraffin embedding, 4- to $5-\mu \mathrm{m}$-thick sections were cut and stained with hematoxylin and eosin.

\section{DNA Isolation and CDKN2X Genotyping}

High MW DNA was extracted from snap-frozen samples of the gills, or from preserved (w/95\% ethyl alcohol) tailfin samples using a Puregene kit (Gentra Systems, Minneapolis, Minnesota). A PCR-based assay was used to determine CDKN2X genotypes of backcross progeny. Primers P16F8 (5'TAAACCAGAACAACTAAGTGG-3') and P16R16 (5'CTGTATTGCTCTTCGTCCA-3') were specifically designed to amplify the $5^{\prime}$ flanking and exon 1 regions of 
the CDKN2X gene. The $5^{\prime}$ flanking region contains a variable copy number GT-repeat that is highly polymorphic between species, enabling quick and reliable allele-specific genotyping. The PCR conditions conformed to those previously published (Kazianis et al, 1998). Amplification from $X$. maculatus (Jp 163 A) resulted in a 1220-bp product, whereas a 1079-bp band was derived from $X$. couchianus. PCR products were generally run on $1.8 \%$ agarose gels, stained with ethidium bromide, and visualized with an IF-500 Gel Documentation System (Alpha Innotech, San Leandro, California).

\section{Acknowledgements}

We gratefully acknowledge the excellent technical assistance of Luis Della Coletta, Leona Hazlewood, Richard Hulsey, Anthony Pedroza, and the histology service core (Science Park Research Division, M. D. Anderson Cancer Center). We especially thank the Xiphophorus Genetic Stock Center of Southwest Texas State University for the various fish strains used.

\section{References}

Ahuja MR, Schwab M, and Anders F (1980). Linkage between a regulatory locus for melanoma cell differentiation and an esterase locus in Xiphophorus. J Hered 71:403-407.

Chin L, Merlino G, and DePinho RA (1998). Malignant melanoma: Modern black plague and genetic black box. Genes Dev 12:3467-3481.

Fornzler D, Wittbrodt J, and Schartl M (1991). Analysis of an esterase linked to a locus involved in the regulation of the melanoma oncogene and isolation of polymorphic marker sequences in Xiphophorus. Biochem Genet 29:509-524.

Frei JV and Lawley PD (1980). Thymomas induced by simple alkylating agents in C57BL/Cbi mice: Kinetics of the dose response. J Natl Cancer Inst 64:845-856.

Friedberg EC, Walker GC, and Siede W (1995). DNA repair and mutagenesis. Washington, DC: ASM Press, 59-83.

Gordon M (1931a). Hereditary basis of melanosis in hybrid fishes. Am J Cancer 15:1495-1523.

Gordon M (1931b). Morphology of the heritable color patterns in the Mexican killifish, Platypoecilus. Am J Cancer 15:732-787.

Joshi VV and Frei JV (1970). Effects of dose and schedule of methylnitrosourea on incidence of malignant lymphoma in adult female mice. J Natl Cancer Inst 45:335-339.

Kallman KD (1975). The platyfish, Xiphophorus maculatus. In: King RC, editor. Handbook of genetics. New York: Plenum Press, 81-132.

Kallman KD and Schreibman MP (1971). The origin and possible genetic control of new, stable pigment patterns in the poeciliid fish Xiphophorus maculatus. J Exp Zool 176: 147-168.

Kazianis S, Coletta LD, Morizot DC, Johnston DA, Osterndorff EA, and Nairn RS (2000). Overexpression of a fish CDKN2 gene in a hereditary melanoma model. Carcinogenesis 21:599-605.
Kazianis S, Gutbrod H, Nairn RS, McEntire BB, Della Coletta L, Walter RB, Borowsky RL, Woodhead AD, Setlow RB, Schartl M, and Morizot DC (1998). Localization of a CDKN2 gene in linkage group $\mathrm{V}$ of Xiphophorus fishes defines it as a candidate for the DIFF tumor suppressor. Genes Chromosomes Cancer 22:210-220.

Kazianis S, Morizot DC, Coletta LD, Johnston DA, Woolcock B, Vielkind JR, and Nairn RS (1999). Comparative structure and characterization of a CDKN2 gene in a Xiphophorus fish melanoma model. Oncogene 18:5088-5099.

Kosswig C (1927). Uber bastarde der teleostier Platypoecilus und Xiphophorus. Z Indukt Abstamm Ver 44:253.

Morizot DC and Siciliano MJ (1983). Linkage group V of platyfishes and Swordtails of the genus Xiphophorus (Poeciliidae): Linkage of loci for malate dehydrogenase-2 and esterase- 1 and esterase- 4 with a gene controlling the severity of hybrid melanomas. J Natl Cancer Inst 71:809-813.

Mullis K, Faloona F, Scharf S, Saiki R, Horn G, and Erlich H (1986). Specific enzymatic amplification of DNA in vitro: The polymerase chain reaction. Cold Spring Harb Symp Quant Biol 51:263-273.

Nairn RS, Kazianis S, McEntire BB, Della Coletta L, Walter $\mathrm{RB}$, and Morizot DC (1996a). A CDKN2-like polymorphism in Xiphophorus LG V is associated with UV-B-induced melanoma formation in platyfish-swordtail hybrids. Proc Natl Acad Sci USA 93:13042-13047.

Nairn RS, Morizot DC, Kazianis S, Woodhead AD, and Setlow RB (1996b). Nonmammalian models for sunlight carcinogenesis: Genetic analysis of melanoma formation in Xiphophorus hybrid fish. Photochem Photobiol 64:440-448.

Ohshima M and Ward JM (1984). Promotion of $N$-methyl- $N$ nitrosourea-induced thyroid tumors by iodine deficiency in F344/NCr rats. J Natl Cancer Inst 73:289-296.

Reed HD and Gordon M (1931). The morphology of melanotic over-growths in hybrids of Mexican killifishes. Am J Cancer 15:1524-1551.

Richie ER, Angel JM, and Rinchik EM (1996). Tlag1, a novel murine tumor susceptibility gene that regulates MNUinduced thymic lymphoma development. Prog Clin Biol Res 395:23-32.

Ruas $M$ and Peters G (1998). The p16INK4a/CDKN2A tumor suppressor and its relatives. Biochim Biophys Acta 1378: F115-F177.

Schartl A, Malitschek B, Kazianis S, Borowsky R, and Schartl $M$ (1995). Spontaneous melanoma formation in nonhybrid Xiphophorus. Cancer Res 55:159-165.

Schartl M (1995). Platyfish and swordtails: A genetic system for the analysis of molecular mechanisms in tumor formation. Trends Genet 11:185-189.

Schwab M, Abdo S, Ahuja MR, Kollinger G, Anders A, Anders F, and Frese K (1978a). Genetics of susceptibility in the platyfish/swordtail tumor system to develop fibrosarcoma and rhabdomyosarcoma following treatment with $N$-methyl- $N$-nitrosourea (MNU). Cancer Res Clin Oncol 91:301-315.

Schwab M, Haas S, Ahuja MR, Kollinger G, Anders A, and Anders F (1978b). Genetic basis of susceptibility for development of neoplasms following treatment with $N$-methyl- $N$ nitrosourea (MNU) or $\mathrm{x}$-rays in the platyfish/swordtail system. Experientia 34:780-782. 
Schwab M, Kollinger G, Haas J, Ahuja MR, Abdo S, Anders A, and Anders F (1979). Genetic basis of susceptibility for neuroblastoma following treatment with $N$-methyl- $N$ nitrosourea and X-rays in Xiphophorus. Cancer Res 39: 519-526.

Setlow RB, Grist E, Thompson K, and Woodhead AD (1993). Wavelengths effective in induction of malignant melanoma. Proc Natl Acad Sci USA 90:6666-6670.

Setlow RB, Woodhead AD, and Grist E (1989). Animal model for ultraviolet radiation-induced melanoma: Platyfish-swordtail hybrid. Proc Natl Acad Sci USA 86: 8922-8926.

Siciliano MJ, Morizot DC, and Wright DA (1976). Factors responsible for platyfish-swordtail hybrid melanomas - many or few? In: Riley V, editor. Pigment cell. Basel: S. Karger, 47-58.

Siciliano MJ and Wright DA (1976). Biochemical genetics of the platyfish-swordtail hybrid melanoma system. Prog Exp Tumor Res 20:398-411.
Sobel HJ, Marquet E, Kallman KD, and Corley GJ (1975). Melanomas in platy/swordtail hybrids. In: Ribelin WE and Migaki G, editors. The pathology of fishes. Madison: The University of Wisconsin Press, 945-981.

Vielkind JR, Kallman KD, and Morizot DC (1989). Genetics of melanomas in Xiphophorus fishes. J Aquat Anim Health 1:69-77.

Vielkind U (1976). Genetic control of cell differentiation in platyfish-swordtail melanomas. J Exp Zool 196:197-204.

Zarbl H, Sukumar S, Arthur AV, Martin-Zanca D, and Barbacid $M$ (1985). Direct mutagenesis of Ha-ras-1 oncogenes by $N$-nitroso- $N$-methylurea during initiation of mammary carcinogenesis in rats. Nature 315:382-385. 